\title{
DOES MOTION PERSPECTIVE INDEPENDENTLY PRODUCE THE IMPRESSION OF A RECEDING -SURFACE? 1
}

\author{
JAMES J. GIBSON AND WALTER CAREL, \\ Cornell University
}

Motion parallax as a fact of optics has been known for a long time. When an observer moves, the apparent relative motion of two or more stationary objects in the visual field is an indicator or clue to their relative distance. The theory that tridimensional perception depends on the interpretation of bidimensional sensations specifies a list of such clues or cues for depth and distance, and motion parallax is an important item on this list. Helmholtz argued that during locomotion, for instance, "the apparent angular velocities of objects in the field of view will be inversely proportional to their real distances away; and consequently safe conclusions can be drawn as to the real distance of the body" $(\mathbf{5}$, p. 295). The reason why these sensations of velocity are experienced as distance rather than as velocity is that the process of interpretation or inference has become so habitual as to become unconscious. We learn to see relative motion as relative distance during the course of growing up in the environment. However, this perception is never more than a "safe conclusion." As Helmholtz was careful to point out, the cues for depth are not compelling for the impression of depth; they function as such only when experience makes the assumption of a third dimension reasonable.

The writer has suggested that this theory is formulated only for objects in space, and that the background of

${ }^{1}$ The experiment here reported was carried out by Walter Carel under the general supervision of the senior author. The study is part of a research project performed under Contract AF 41(128) 42 between Cornell University and the USAF School of Aviation Medicine. objects-the ground or terrain-is left out of account (3, ch. 7). When the retinal image is considered as the projection of a continuous array of textured surfaces, it becomes possible to state the fact of motion perspective more generally, as deformation or skew in the retinal image, and to analyze the gradients of velocity and direction. A more parsimonious theory then becomes possible by assuming that the velocity gradient for the elements of a surface is a stimulus for the impression of distance. Phenomenal distance is conceived as the recession of a surface, or an array of surfaces, rather than as the optical third dimension.

The decrease in "crossed" velocity in the visual field with the increase in distance may be termed motion-perspective, by analogy with the increase in the density of contours with increasing distance $(3$, p. $137 \mathrm{ff}$.). Size-perspective, texture-perspective, and linear perspective are all special cases of the rule that density in the visual field goes with recession in the visual world. The perspective of crossed diplopia usually accompanies the perspective of crossed velocity, but it is absent in monocular vision.

The experimental study of motionperspective (as distinguished from the motion parallax of isolated objects in empty space) requires that adequate stimulation be present for the impression of an extended surface, i.e., a differentiated retinal image (4). A difficulty is then faced: how can $E$ isolate motion-perspective from density-perspective? If a gradient of velocity in the retinal image is set up with an even distribution of elements, this 
constitutes a zero gradient of density tending to produce a frontal surface which conflicts with the expected receding surface. If a gradient of velocity is set up with a congruent gradient of density, then the former has not been isolated. The latter case holds for the motion-picture methods of presenting velocity-gradients tried out during the last war, notably in the so-called "Landing Judgment Test" (2, ch. 9).

The conclusion seems to be that motion-perspective cannot be isolated from density-perspective, properly speaking, but can only be put in opposition to it. In the face of this difficulty, it is nevertheless important to discover whether an "elastic deformation" of the retinal image will overcome an even distribution of elements to produce the impression of a receding surface.

\section{Method}

It was decided that the source of stimulation should be a bank of scattered points of light in a dark field of view, without alignment in rows and having a uniform over-all density. When motionless, this arrangement should produce an impression of spots on a frontal plane. The lights, however, should be capable of moving across the field, appearing on one side and disappearing on the other, with a velocity decreasing upward from the bottom of the bank to the top. With respect to velocity, the view would be similar to that produced at night by looking out the window of an airplane at the lights of a city. The difference would be that the city lights would also have a gradient of increasing density and decreasing size. The question to be answered was whether $O$ would see an array of spots in the frontal plane when the lights were motionless but a plane receding upward when they were moved. Along with the latter impression there might be a feeling that $O$ himself was moving.

Procedure.-The device constructed to produce this effect was a 6 -ft. black disk mounted upright with a bearing at its center around which it could be rotated. Only a sector at the bottom of the disk would be visible at any one time. Its surface was entirely covered with narrow lines of luminous paint radiating outward from the center. The lines were broken segments, however, rather than continuous radii and these line-seg- ments 6-8 in. long were spaced about as far apart at the periphery as at the center. In front of the disk was a screen, opaque in all parts except for a triangular area, base down, in which thin horizontal slits were cut, parallel and about an inch apart. A sector of the luminous lines was thus permitted to shine through the horizontal slits, making a roughly triangular field of points. The random distribution of the radiating segments prevented any alignment of the points such as to suggest linear perspective. The luminous paint was activated by a "black light" lamp, but otherwise the room was in darkness.

Rotation of the disk produced velocities of the spots approximately proportional to their distance from the center, i.e., a gradient of velocities. The velocity of any spot was not, however, quite uniform along its horizontal slit, being greater at the beginning and end of the path. For the case of motion-perspective as observed through the window of a plane or train, the velocity of a point is greater at the middle of the path. The motion-perspective obtained by the device, therefore, was only approximately that of a level terrain projected on a picture-plane.

Each $O$ was allowed some minutes for darkadaptation and then the motionless bank of spots was presented to one eye, at approximately the distance of the theorctical station-point. The line of sight was horizontal and the field occupied $30^{\circ}-40^{\circ}$ of visual angle. A report of the perception was then obtained. The disk was next set in rotation and the experience was again described. One group of $7 \mathrm{Os}$ was made up of the $i s$ themselves and other experienced $O$ s who understood the apparatus and knew the purpose of the experiment. A second group of $100 \mathrm{~s}$ was obtained who were ignorant of both.

\section{Results}

All $O$ s saw the motionless bank of lights in a plane perpendicular to the eye. No experience of slant, recession, or increasing distance was reported. When the lights were in motion, the "sophisticated" $O$ s could see the spots progressively more distant toward the top, in a plane which receded. There were even reports of subjective motion in the direction opposite that of the lights. These descriptions are worth little, however, since they might have been influenced by expectation or suggestion. Only the spontaneous reports of the "naive" $O_{s}$ can be relicd on, and these were 
quite different. Six of the ten saw a group of isolated spots, some of which were moving faster than others, all at the same distance from the eye. The spots appeared to be separate objects, moving independently. Three of the ten reported something like the light of a city at night, with a receding plane of distance. One was wholly equivocal.

\section{Conclusions}

The results of the experiment were negative. No compelling or universally obtained impression of recession, slant, or relative distance was induced by the skewed motion of the bank of lights, and even less evident was any impression that the $O$ himself moved.

It was recognized at the outset that a bank of luminous points might not produce adequate stimulation for a visual surface. Small spots separated by large areas of darkness are quite different from the adjacent highlights and shadows of a texture. The bank of lights did not seem to have much "hardness" or "impenetrability"; there was an impression that one could see through them (4).

The majority of $O$ s saw the lights as a collection of discrete objects in empty space, not as elements linked together in a surface. It seems likely that one property of a surface is phenomenal rigidity during all kinds of motion, and this property was weak or absent in the banks of lights.

The conclusion must be that under the conditions of this experiment the moving spots functioned truly as clues or cues for distance rather than as stimuli. As Helmholtz himself recognized, the cues for depth are not compelling or determining since attitudes, assumptions, or inferences must enter into the perceptual process. Motion parallax, then, can be perceived either as differences in velocity without differences in depth or differences in depth without differences in velocity. When the elements of the retinal image are separated points of light, and when the gradient of their motion is incompatible with the gradient of their density, the resulting perceptions of depth are ambiguous.

The fact that it is not possible to isolate a gradient of motion from a gradient of density but only to produce one which is incongruent with the other illustrates once more the primary status of the density variable in the retinal image for spatial impressions. Only when the image is composed of sharply differentiated regions (4), can the perspective gradients exist. The three main space-determining gradients (density, motion, and binocular disparity) might conceivably be related in this way: that only when they are congruent are they psychophysically determining; when incongruent, they serve merely as clues for probable inferences (1).

(Manuscript received September 24, 1951)

\section{REFERENCES}

1. Brunswik, E. Distal focussing of perception. Psychol. Monogr., 1944, 56, No. 1 (whole no. 254).

2. Grason, J. J. (Ed.) Motion picture testing and research. Washington: U. S. Govt. Printing Office, 1947.

3. Greson, J. J. The perception of the visual vorld. Boston: Houghton Miflin, 1950.

4. Gibson, J. J., \& Dibbie, F. N. Exploratory experiments on the stimulus conditions for the perception of a visual surface. $J$. exp. Psychol., 1952, 43, 414-419.

5. Helmholtz, H. Physiological optics, Vol. 3. (Ed. J. P. C. Southall) New York: Opt. Soc. Amer., 1925. 